\title{
On the algebraic K-theory of formal power series
}

\author{
by \\ Ayelet Lindenstrauss And RANDy McCARThy
}

\begin{abstract}
In this paper we extend the computation of the the typical curves of algebraic K-theory done by Lars Hesselholt and Ib Madsen to general tensor algebras. The models used allow us to determine the stages of the Taylor tower of algebraic K-theory as a functor of augmented algebras, as defined by Tom Goodwillie, when evaluated on derived tensor algebras.

For $R$ a discrete ring, and $M$ a simplicial $R$-bimodule, we let $\mathcal{T}_{R}(M)$ denote the (derived) tensor algebra of $M$ over $R$, and $\mathcal{T}_{R}^{\pi}(M)$ denote the ring of formal (derived) power series in $M$ over $R$. We define a natural transformation of functors of simplicial $R$-bimodules $\Phi: \Sigma \tilde{K}(R ;) \rightarrow \tilde{K}\left(\mathcal{T}_{R}^{\pi}()\right)$ which is closely related to Waldhausen's equivalence $\Sigma \tilde{K}(\operatorname{Nil}(R ;)) \stackrel{\simeq}{\rightarrow} \tilde{K}\left(\mathcal{T}_{R}()\right)$. We show that $\Phi$ induces an equivalence on any finite stage of Goodwillie's Taylor towers of the functors at any simplicial bimodule. This is used to show that there is an equivalence of functors $\Sigma W(R ;) \stackrel{\simeq}{\rightarrow} \operatorname{holim}_{n} \tilde{K}\left(\mathcal{T}_{R}() / I^{n+1}\right)$, and for connected bimodules, also an equivalence $\Sigma \tilde{K}(R ;) \stackrel{\simeq}{\rightarrow} \tilde{K}\left(\mathcal{T}_{R}()\right)$.
\end{abstract}

Key Words: Algebraic K-theory, K-theory of Endomorphisms, Goodwillie Calculus, Formal Power Series, Tensor Algebra

Mathematics Subject Classification 2000: 19D50 ; 19D35, 19D55, 16E20

\section{Introduction}

Throughout this paper, $R$ denotes a discrete unital ring, and $M$ a simplicial $R$ bimodule. We let $K(R ; M)$ be the algebraic K-theory of parametrized (in $M$ ) endomorphisms, which is the K-theory of the category whose objects are pairs $(P, f)$ with $P$ a finitely generated projective right $R$-module and $f: P \rightarrow P \otimes_{R} M$ a map of right $R$ modules, and whose maps are maps of the modules $P$ which induce commutative diagrams. Note that $K(R ; 0)=K(R)$ is a retract of $K(R ; M)$ for any bimodule $M$; we label the remaining part $\tilde{K}(R ; M)=\operatorname{hofib}(K(R ; M) \rightarrow K(R ; 0))$.

We can look at the tensor algebra (with derived tensor products) of $M$ over $R$,

$$
\mathcal{T}_{R}(M)=R \oplus M \oplus M \otimes_{R}^{\wedge} M \oplus M \otimes_{R}^{\wedge} M \otimes_{R}^{\wedge} M \oplus \cdots .
$$

If $M$ is an $R$-bimodule which is flat either as a right $R$-module or as a left $R$-module, then this is just the usual tensor algebra $T_{R}(M)$. The algebra $\mathcal{T}_{R}(M)$ is augmented 
over $R$; if $I$ is its augmentation ideal, then the formal power series in $M$ over $R$ are $\mathcal{T}_{R}^{\pi}(M)=\lim _{n} \mathcal{T}_{R}(M) / I^{n+1}$.

The main result of this paper, Theorem 3.1, says that a particular natural transformation of functors of simplicial $R$-bimodules that we construct,

$$
\Phi: \Sigma \tilde{K}(R ;) \rightarrow \tilde{K}\left(\mathcal{T}_{R}^{\pi}()\right),
$$

induces an equivalence on the $n$ 'th stage of Goodwillie's Taylor tower, $\mathbf{P}_{n}$, of the functors for every $n$. If a bimodule $M$ is connected, then the map $\mathcal{T}_{R}(M) \rightarrow \mathcal{T}_{R}^{\pi}(M)$ is an equivalence, and both Goodwillie Taylor towers converge to the functors that they are approximating. Thus, Theorem 3.1 gives Corollary 3.3, which says that for connected $R$-bimodules $M$,

$$
\Phi: \Sigma \tilde{K}(R ; M) \stackrel{\simeq}{\rightarrow} \tilde{K}\left(\mathcal{T}_{R}(M)\right) .
$$

For a general bimodule $M$, we do not have convergence of Goodwillie's Taylor towers. However, we can readily describe what Goodwillie's Taylor tower of $\tilde{K}(R ; M)$ actually is: it is an invariant called $W(R ; M)$ which we will describe below. Also, we can compare $\tilde{K}\left(\mathcal{T}_{R}^{\pi}()\right)$ to the more tractable functors $\tilde{K}\left(\mathcal{T}_{R}() / I^{n+1}\right)$, whose Goodwillie Taylor towers do converge to them. We obtain as Corollary 3.2 the formula

$$
\Sigma W(R ;) \simeq \operatorname{holim}_{n} \tilde{K}\left(\mathcal{T}_{R}() / I^{n+1}\right) .
$$

Note that the results of Corollaries 3.3 and 3.2 are absolute results, that is-they do not require pro-finite completion.

The idea for the map $\Phi$ that we use came from Waldhausen [W1], where he defines an equivalence

$$
\Sigma \tilde{K}(\operatorname{Nil}(R ;)) \stackrel{\simeq}{\rightarrow} \tilde{K}\left(\mathcal{T}_{R}()\right) .
$$

One can model $K(\operatorname{Nil}(R ; M)$ ) (see also [B]) as the algebraic K-theory of the full subcategory of the category we used to define $K(R ; M)$ consisting of modules $P$ and maps $m: P \rightarrow P \otimes_{R} M$ which are nilpotent, that is, for every $p \in P$ some power $m^{\otimes_{R} i}$ vanishes on $p$ (see equation (3.4) below for the meaning of $m^{\otimes_{R} i}$ ). In these terms, for a nilpotent map $m: P \rightarrow P \otimes_{R} M$, Waldhausen's equivalence sends

$$
m \mapsto(1-m)^{-1}=\Sigma_{i=0}^{\infty} m^{\otimes_{R} i}
$$

where the latter is extended $\mathcal{T}_{R}(M)$-linearly to be viewed as a map from $P \otimes_{R}$ $\mathcal{T}_{R}(M)$ to itself, and the infinite sum makes sense because at every point in the 
domain of the map, the infinite sum is in fact finite. This suggests that it would be interesting to look at this map $m \mapsto(1-m)^{-1}$ defined on the full $\tilde{K}(R ; M)$. Of course, the map would not be able to land in $\tilde{K}\left(\mathcal{T}_{R}(M)\right)$ anymore because of the problem of the convergence of the infinite sum, but would land in some sort of (noncommutative, unless $R$ is commutative and $M$ is symmetric with a single generator) localization of it which inverts elements of the form $1-m$ from $P \otimes_{R} \mathcal{T}_{R}(M)$ to itself, and the idea would be that one would get a commutative diagram

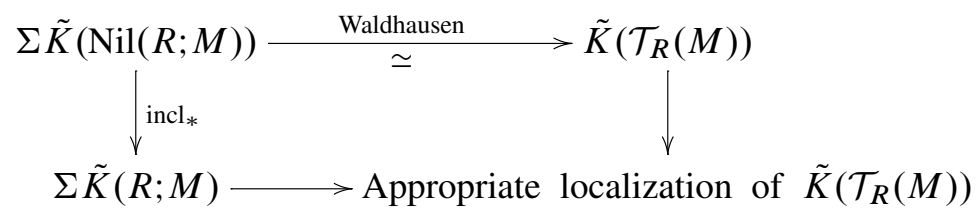

with the bottom horizontal map a weak equivalence as well. For connected $M$, our map $\Phi$ does exactly that, and no localization is even required since the elements $1-m$ are 'homotopically invertible'. In the general case, we can define a bottom horizontal $\Phi$ if we replace $\tilde{K}\left(\mathcal{T}_{R}(M)\right)$ by $\tilde{K}\left(\mathcal{T}_{R}^{\pi}(M)\right)$, but it only induces an equivalence on Goodwillie's Taylor towers.

Betley [B] began the program of realizing the square (1.1) with horizontal equivalences by showing that when $R$ is a field and $M$ a discrete $R$-bimodule, the invariant we call $\tilde{K}(R ; M)$ is a localization in the sense of Neeman and Ranicki [NR] of $\Omega \tilde{K}\left(T_{R}(M)\right)$, coming from inverting maps of the form $1-m$ in the category of finitely generated projective $T_{R}(M)$ modules (since $R$ is a field, there is no need to take the derived tensor algebra). In recent work of Klein and Williams, they are able to realize the square (1.1) with horizontal equivalences when $M$ is equal to $R$ itself with twisted multiplication, that is, with the standard $R$-action on the left but with action via some endomorphism of $R$ on the right. They use a derived noncommutative Dwyer localization at the ring level on $T_{R}(M)$ (again there is no need to take the derived tensor algebra since $M$ is free as a left $R$-module).

The invariant $W(R ; M)$ comes from the calculation of Goodwillie's Taylor tower of the K-theory of parametrized endomorphisms in [LMcC1]: we look at circular derived tensor products of $i$ copies of $M$,

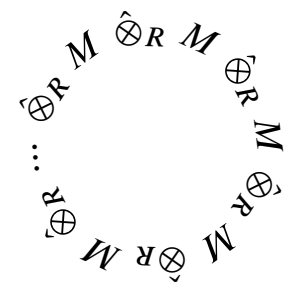


where the cyclic group $C_{i}$ acts by rotation; we call this invariant $U^{i}(R ; M)$ (so $U^{1}(R ; M)$ is the same thing as $\left.\operatorname{THH}(R ; M)\right)$. The $n$ 'th stage of Goodwillie's Taylor tower of $M \mapsto \tilde{K}(R ; M)$, denoted $\mathbf{P}_{n} \tilde{K}(R ; M)$, is $\operatorname{holim}_{i \leq n} U^{i}(R ; M)^{C_{i}}$, where the homotopy inverse limit is taken over restriction maps $U^{j}(R ; M)^{C_{j}} \rightarrow U^{i}(R ; M)^{C_{i}}$ defined whenever $i$ divides $j$; we call this $W_{n}(R ; M)$. We also let $W(R ; M)=$ $\operatorname{holim}_{i \in \mathbb{N} \times} U^{i}(R ; M)^{C_{i}}=\operatorname{holim}_{n} W_{n}(R ; M)$, with limits taken over the restriction maps; this is the full Goodwillie Taylor tower. When $M=R, W(R ; R)$ was used as an intermediate stage in defining TC $(R)$ in [BHM], and was called TR $(R)$ in [HM].

Another motivation for the result of Corollary 3.3 comes from [CCGH], where Carlsson et al. show in Theorem 3 that for a simplicial space $X$,

$$
A(\Sigma X) \simeq \Sigma \bigvee_{n=1}^{\infty}\left[(\mathbb{S} X)^{\wedge n}\right]_{h C_{n}} .
$$

But Milnor shows in Theorem 5 of [M] that for connected $X$, there is an equivalence $\Sigma \Omega \Sigma X \simeq \bigvee_{i=1}^{\infty} \Sigma X^{\wedge i}$ which respects the maps induced by loop concatenation and smashing copies of $X$, and thus induces an equivalence $\Sigma^{\infty}\left((\Omega \Sigma X)_{+}\right) \simeq T_{\mathbb{S}} \mathbb{S} X$ (one can use the tensor algebra $T_{\mathbb{S}} \mathbb{S} X$ rather than the derived one $\mathcal{T}_{\mathbb{S}} \mathbb{S} X$ since $\mathbb{S} X$ is cellular over $\mathbb{S}$ ). Therefore, for $X$ connected

$$
A(\Sigma X)=K\left(\Sigma^{\infty}\left((\Omega \Sigma X)_{+}\right)\right) \simeq K\left(T_{\mathbb{S}} \mathbb{S} X\right),
$$

while tom Dieck splitting (as in [BHM] for the case $R=M$, and more generally as in [I]) gives $\Sigma W(\mathbb{S}, \mathbb{S} X) \simeq \Sigma \bigvee_{n=1}^{\infty}\left[(\mathbb{S} X)^{\wedge n}\right]_{h C_{n}}$. In these terms, then, the [CCGH] result could be written for connected $X$ as

$$
\Sigma W(\mathbb{S}, \mathbb{S} X) \simeq K\left(T_{\mathbb{S}} \mathbb{S} X\right),
$$

which is a version for functors with smash product (which we do not prove-we only look at discrete rings $R$ and their simplicial bimodules $M$ ) of our result of Corollary 3.3 for $R=\mathbb{S}$ and $M=\mathbb{S} X$.

Reading in the opposite direction, if we are interested in understanding the Ktheory of augmented simplicial $R$-algebras rather than the K-theory of endomorphisms, the equation

$$
\Phi_{n}: \Sigma W_{n}(R ;) \stackrel{\simeq}{\rightarrow} \mathbf{P}_{n} \tilde{K}\left(\mathcal{T}_{R}^{\pi}()\right)
$$

(where $\mathbf{P}_{n}$ is the $n$ 'th stage of Goodwillie's Taylor tower) which is proved in Theorem 3.1 (applied to connected $M$, for which $\mathcal{T}_{R}^{\pi}(M) \simeq \mathcal{T}_{R}(M)$ ) tells us 
the finite stages of Goodwillie's Taylor tower of the functor $M \mapsto \tilde{K}\left(\mathcal{T}_{R}(M)\right)$ on simplicial $R$-bimodules (since the stages of Goodwillie's Taylor tower are determined by their values on connected spaces).

It would be interesting to know the stages of Goodwillie's Taylor tower of the functor $A \mapsto \tilde{K}(A)$ on the category of augmented simplicial $R$-algebras. While we cannot deduce this directly from our result, it is interesting to note that we have determined these stages when applied to tensor algebras. In general, Goodwillie's Taylor tower of a functor $F$ from augmented simplicial $R$-algebras to spectra, applied to (derived) tensor algebras of the form $\mathcal{T}_{R}(M)$, coincides with Goodwillie's Taylor tower of the functor $F\left(\mathcal{T}_{R}()\right)$ from simplicial $R$-bimodules to spectra when applied to $M$. This is because the functor $M \mapsto \mathcal{T}_{R}(M)$ from $R$-bimodules to augmented $R$-algebras sends the initial and final object 0 to the initial and final object $R$, coproducts to coproducts, and more generally: coCartesian cubes to co-Cartesian cubes. Recall (see [G3]) that Goodwillie constructs $\mathbf{P}_{n} F(X)=\operatorname{hocolim}_{i}\left(T_{n}^{i} F\right)(X)$, and the iterated maps $T_{n}$ involve taking homotopy limits of the functor in question over co-Cartesian diagrams of coproducts of $F(X)$ with the initial and final object, so this construction would be the same for $F$ on $R$ algebras and for $F\left(\mathcal{T}_{R}()\right)$ on $R$-bimodules. Therefore, in this paper we determine the values that the finite Goodwillie Taylor approximations $\mathbf{P}_{n} \tilde{K}$ take on augmented $R$-algebras which are of the form $\mathcal{T}_{R}(M)$.

For $M=R$, Grayson [Gr] showed in 1977, at least at the level of homotopy groups, that the K-theory of endomorphisms

$$
\Sigma K(R ; R) \simeq K\left((1+x R[x])^{-1} R[x]\right),
$$

where the localization is straightforward because it is done on the level of the underlying commutative ring, and of course $R[x]$ is the same thing as $T_{R}(R)$. In the case of $M=R$, our result of Corollary 3.2,

$$
\Sigma \mathrm{TR}(R) \simeq \operatorname{holim}_{n} \tilde{K}\left(R[x] /(x)^{n+1}\right),
$$

is new, but the fact that it is true after pro-finite completion was proved by Hesselholt $[\mathrm{H}]$ in the commutative case, and follows from the work of Betley and Schlichtkrull in [BS] for general $R$. It is possible to recover our result in the $M=R$ case from the $[\mathrm{H}]$ and $[\mathrm{BS}]$ results by an arithmetic square argument using the work of Goodwillie in $[\mathrm{G}]$.

When what came to be called TR $(R)$ was used in [BHM] (see Definition 5.12 there), it was as an intermediate stage in the calculation of $\operatorname{TC}(R)$. The definition of $\mathrm{TC}(R)$ is the homotopy inverse limit of $\operatorname{sd}_{i} \operatorname{THH}(R)^{C_{i}}$ (which is the same as 
$\left.U^{i}(R ; R)^{C_{i}}\right)$ taken over two kinds of maps, restriction maps like those we look at and also Frobenius maps (which are simply inclusions of the fixedpoints of $C_{j}$ into the fixedpoints of a subgroup $C_{i}$ if $i$ divides $j$ ). When $M$ is connected, the cyclotomic trace of $[\mathrm{BHM}]$ gives, by $[\mathrm{McC}]$,

$$
\tilde{K}\left(\mathcal{T}_{R}(M)\right)_{p}^{\wedge} \stackrel{\sim}{\rightarrow} \operatorname{hofib}\left(\operatorname{TC}\left(\mathcal{T}_{R}(M)\right) \rightarrow \operatorname{TC}(R)\right)_{p}^{\wedge}
$$

at any prime $p$ : this is because since $M$ is connected, $\mathcal{T}_{R}(M)=\lim _{n} \mathcal{T}_{R}(M) / I^{n+1}$, and since the maps $\mathcal{T}_{R}(M) \rightarrow \mathcal{T}_{R}(M) / I^{n+1}$ become increasingly connected, we get $\tilde{K}\left(\mathcal{T}_{R}(M)\right) \simeq \operatorname{holim}_{m} \tilde{K}\left(\mathcal{T}_{R}(M) / I^{n+1}\right)$, where the $\mathcal{T}_{R}(M) / I^{n+1}$ have nilpotent augmentation ideals as required by the $[\mathrm{McC}]$ result. We can let $\tilde{\mathrm{TC}}\left(\mathcal{T}_{R}(M)\right)=$ $\operatorname{hofib}\left(\mathrm{TC}\left(\mathcal{T}_{R}(M)\right) \rightarrow \operatorname{TC}(R)\right)$ and $\tilde{\mathrm{TR}}\left(\mathcal{T}_{R}(M)\right)=\operatorname{hofib}\left(\operatorname{TR}\left(\mathcal{T}_{R}(M)\right) \rightarrow \operatorname{TR}(R)\right)$.

To relate between (1.2) and the result of Corollary 3.3 here which can be rewritten as

$$
\Sigma W(R ; M) \stackrel{\simeq}{\rightarrow} \tilde{K}\left(\mathcal{T}_{R}(M)\right),
$$

we start by using the cofibration $S^{0} \rightarrow S_{+}^{1} \rightarrow S^{1}$ whose first map has a basepoint preserving splitting to send

$$
\Sigma W(R ; M)=S^{1} \wedge W(R ; M) \rightarrow S_{+}^{1} \wedge W(R ; M) .
$$

Then we use the obvious inclusions $R \hookrightarrow \mathcal{T}_{R}(M)$ and $M \hookrightarrow \mathcal{T}_{R}(M)$ to map

$$
S_{+}^{1} \wedge W(R ; M) \rightarrow S_{+}^{1} \wedge W\left(\mathcal{T}_{R}(M) ; \mathcal{T}_{R}(M)\right)=S_{+}^{1} \wedge \operatorname{TR}\left(\mathcal{T}_{R}(M)\right) .
$$

Since the obvious inclusion sends $M$ into the augmentation ideal of $\mathcal{T}_{R}(M)$, this actually lands in $S_{+}^{1} \wedge \tilde{\mathrm{TR}}\left(\mathcal{T}_{R}(M)\right)$. But TR of any ring has a basepoint-fixing $S^{1}$ action inherited from that of THH which is a cyclic spectrum: this is because we can make the restriction maps be $S^{1}$-maps. Moreover this action preserves TR $(R)$ so defines an action on TR as well. This gives an action map

$$
S_{+}^{1} \wedge \tilde{\mathrm{TR}}\left(\mathcal{T}_{R}(M)\right) \rightarrow \tilde{\mathrm{TR}}\left(\mathcal{T}_{R}(M)\right) .
$$

Assembled together, this all gives a map

$$
\tilde{K}\left(\mathcal{T}_{R}(M)\right) \simeq \Sigma W(R ; M) \rightarrow \tilde{\mathrm{TR}}\left(\mathcal{T}_{R}(M)\right),
$$

which is far from being an equivalence. But if we complete at $p$, then $\tilde{\mathrm{TR}}\left(\mathcal{T}_{R}(M)\right)_{p}^{\wedge}$ splits as a product of copies of $\tilde{\mathrm{TR}}^{(p)}\left(\mathcal{T}_{R}(M)\right)$, each corresponding to taking the inverse limit over a subset $\left\{k, k p, k p^{2}, \ldots\right\}$ of $\mathbb{N}^{\times}$where $k$ is coprime to $p$. If one were to then take homotopy inverse limits over the Frobenius maps as well, they would identify the factors belonging to the different $k$ 's into one, and make that factor into $\tilde{\mathrm{TC}}\left(\mathcal{T}_{R}(M)\right)_{p}^{\wedge}$. Applying that process to the map (1.3) would recover the map (1.2). 


\section{Preliminaries}

For $R$ a unital ring and $M$ an $R$-bimodule we can look at the tensor algebra (with derived tensor products) of $M$ over $R$,

$$
\mathcal{T}_{R}(M)=R \oplus M \oplus M \otimes_{R}^{\wedge} M \oplus M \otimes_{R}^{\wedge} M \otimes_{R}^{\wedge} M \oplus \cdots .
$$

Then $\mathcal{T}_{R}(M)$ is an augmented $R$-algebra, and we call its augmentation ideal $I$. Note that if $M$ is an $R$-bimodule which is flat either as a right $R$-module or as a left $R$ module, then the tensoring down map $M^{\otimes_{R^{n}}} \rightarrow M^{\otimes_{R}}$ is a weak equivalence for every $n$. This makes $\mathcal{T}_{R}(M)$ weakly equivalent to the usual tensor algebra for such $M$, which we can denote by $T_{R}(M)$.

We let $\mathcal{P}_{R}$ denote the category of projective finitely generated right $R$-modules, and $\mathcal{M}_{R}$ denote the category of finitely generated right $R$-modules. For an augmented $R$-algebra $A \stackrel{\eta}{\rightarrow} R$ with augmentation ideal $I$ and an element $P \in \mathcal{P}_{R}$, we will set

$$
I_{P}(A)=\operatorname{Hom}_{\mathcal{M}_{R}}\left(P, P \otimes_{R} I\right) \cong \operatorname{ker}\left(\operatorname{Hom}_{\mathcal{M}_{R}}\left(P, P \otimes_{R} A\right) \stackrel{\eta_{*}}{\rightarrow} \operatorname{Hom}_{\mathcal{M}_{R}}(P, P)\right) .
$$

Following the construction in section I.2.5 of [DGMcC] and letting $\mathbf{1}_{P}$ denote the identity element in $\operatorname{Hom}(P, P)$, we can view $\mathbf{1}_{P}+I_{P}(A)$ as a subset of $\operatorname{Hom}_{\mathcal{M}_{R}}\left(P \otimes_{R} A, P \otimes_{R} A\right)$ as follows: for $\alpha \in I_{P}(A)$, let

$$
\left(\mathbf{1}_{P}+\alpha\right)(p \otimes a)=(p \otimes 1+\alpha(p)) a=p \otimes a+\alpha(p) a .
$$

Viewed inside $\operatorname{Hom}_{\mathcal{M}_{R}}\left(P \otimes_{R} A, P \otimes_{R} A\right)$, we can compose elements of $\mathbf{1}_{P}+I_{P}(A)$; applying this to $\mathbf{1}_{P}+\alpha$ and $\mathbf{1}_{P}+\beta$ will give the composition

$$
P \stackrel{1_{P}+\alpha}{\longrightarrow} P \otimes A \stackrel{\left(1_{P}+\beta\right) \otimes 1_{A}}{\longrightarrow} P \otimes A \otimes A \stackrel{1_{P} \otimes \text { mult }_{A}}{\longrightarrow} P \otimes A .
$$

sending

$$
p \mapsto p \otimes 1+\alpha(p)+\beta(p)+\beta(\alpha(p)),
$$

where $\beta(\alpha(p))$ is interpreted using $A$-linearity as above. Note that if $\alpha, \beta$ send $P$ to $P \otimes I$, so does $\alpha+\beta+\beta(\alpha)$, so $\mathbf{1}_{P}+I_{P}(A)$ is closed under multiplication. If there is some reason that for any $\alpha \in I_{P}(A), \mathbf{1}_{P}+\alpha+\alpha^{2}+\cdots$ is defined (such as the augmentation ideal being nilpotent or the infinite sum converging for another reason), it is in fact a group. We look at its classifying space. We can by $[\mathrm{DGMcC}]$ model the reduced (over $R$ ) K-theory spectrum $\tilde{K}(A)$ using Waldhausen's S-construction

$$
\left\{n \mapsto \bigvee_{P \in S^{(n)} \mathcal{P}_{R}} B .\left(\mathbf{1}_{P}+I_{P}(A)\right)\right\}
$$


We will be looking at the augmented $R$-algebras $\mathcal{T}_{R}(M) / I^{n+1}$ and

$$
\mathcal{T}_{R}^{\pi}(M)=\lim _{n} \mathcal{T}_{R}(M) / I^{n+1} .
$$

They both satisfy the condition that for any $P \in \mathcal{P}_{R}$ and $\alpha \in I_{P}(A), \mathbf{1}_{P}+\alpha+\alpha^{2}+\cdots$ is defined in $\operatorname{Hom}_{R}\left(P, P \otimes_{R} A\right)$.

Proposition 2.1 The functor $M \mapsto \tilde{K}\left(\mathcal{T}_{R}(M) / I^{n+1}\right)$, as a functor from simplicial R-bimodules to spectra,

1) commutes with realizations

2) satisfies the colimit axiom, that is: respects filtered colimits

3) preserves connectivity of maps

4) is -1-analytic

so $\tilde{K}\left(\mathcal{T}_{R}(M) / I^{n+1}\right)=\operatorname{holim}_{k} \mathbf{P}_{k} \tilde{K}\left(\left(\mathcal{T}_{R}(M) / I^{n+1}\right)\right.$ for all simplicial $R$-bimodules $M$.

Proof: Condition 1) follows from chapter III of [DGMcC]. Conditions 2) and 3) follow from the facts that by direct observation these properties are true for the functor $M \mapsto \mathcal{T}_{R}(M) / I^{n+1}$ and by [W2] they are true for the algebraic $K$-theory of simplicial rings.

By taking resolutions if necessary and using the colimit axiom, to show 4) it suffices to show that the functor of spaces

$$
X \mapsto \tilde{K}\left(\mathcal{T}_{R}\left(\tilde{R}\left[X_{+}\right]\right) / I^{n+1}\right)
$$

is -1-analytic. To do this we'll follow the process done for the case $n=1 \mathrm{in}[\mathrm{McC}]$ (Proposition 3.2) which is essentially a modification of work by Goodwillie in [G2].

Let $\mathcal{X}$ be a strongly co-Cartesian $S$-cube of spaces. We may assume that the natural maps are inclusions of sub-simplicial sets. Suppose that the maps $\mathcal{X}(\emptyset) \rightarrow$ $\mathcal{X}(\{s\})$ are $k_{s}$-connected for each $s \in S$. We wish to show that the stabilization of the cube of functors

$$
\begin{aligned}
\bigvee_{P \in \mathcal{P}_{R}} B .\left(\mathbf{1}_{P}+I_{P}\left(\mathcal{T}_{R}\left(\tilde{R}\left[\mathcal{X}_{+}\right]\right) / I^{n+1}\right)\right) \\
\\
\cong \bigvee_{P \in \mathcal{P}_{R}} B .\left(\mathbf{1}_{P}+\operatorname{Hom}_{\mathcal{M}_{R}}(P, P) \otimes_{\mathbf{Z}} \tilde{\mathbf{Z}}\left[\mathcal{X}_{+} \vee \mathcal{X}_{+}^{2} \vee \cdots \vee \mathcal{X}_{+}^{n}\right]\right)
\end{aligned}
$$

is $|S|-1+\Sigma k_{s}$ Cartesian, since then the functor $\tilde{K}\left(\mathcal{T}_{R}\left(\tilde{R}\left[X_{+}\right]\right) / I^{n+1}\right)$ will satisfy $E_{|S|-1}(1-|S|)$ and hence be -1 analytic. If we show that the cube 
B. $\left(\mathbf{1}_{P}+I_{P}\left(\mathcal{T}_{R}\left(\tilde{R}\left[\mathcal{X}_{+}\right]\right) / I^{n+1}\right)\right)$ is $2(|S|-1)+\left(\Sigma k_{s}\right)$ co-Cartesian for all $P \in \mathcal{P}_{R}$, then since (homotopy) colimits commute and a $q$-reduced simplicial space of $t$ connected spaces is $(q+t)$-connected, $\bigvee_{P \in S_{\bullet}^{(q)}} \mathcal{P}_{R} B .\left(\mathbf{1}_{P}+I_{P}\left(\mathcal{T}_{R}\left(\tilde{R}\left[\mathcal{X}_{+}\right]\right) / I^{n+1}\right)\right)$ will be $\left(q+2(|S|-1)+\Sigma k_{s}\right)-$ co-Cartesian. By taking $\Omega^{q}$ of these and the limit with respect to $q$ we will obtain a $2(|S|-1)+\Sigma k_{s}$-co-Cartesian diagram of spectra which is equivalent to a $|S|-1+\Sigma k_{s}$-Cartesian diagram of spectra (see [G2], 1.19) and hence the result.

We will prove that in general, for the $S$-cube $\mathcal{X}$

$B .\left(\mathbf{1}_{P}+I_{P}\left(\mathcal{T}_{R}\left(\tilde{R}\left[\mathcal{X}_{+}\right]\right) / I^{n+1}\right)\right) \cong B .\left(\mathbf{1}_{P}+\operatorname{Hom}_{R}(P, P) \otimes_{\mathbf{Z}} \tilde{\mathbf{Z}}\left[\mathcal{X}_{+} \vee \mathcal{X}_{+}^{2} \vee \cdots \vee \mathcal{X}_{+}^{n}\right]\right)$

is $2(|S|-1)+\Sigma k_{s}$ co-Cartesian by induction on $n$. We recall that by Theorem 2.6 of [G2], to show an $S$-cube $\mathcal{Y}$ is $2(|S|-1)+\Sigma k_{s}$-co-Cartesian is suffices to show Induction Hypothesis 2.2 For each $T \neq \emptyset$ the $T$-cube $\partial_{S-T} \mathcal{Y}$ is $2(|T|-1)+$ $\Sigma_{t \in T} k_{t}$-Cartesian.

In proposition 3.2 of $[\mathrm{McC}]$, the case for $n=1$ was done. In particular, the cube B. $\left(\mathbf{1}_{P}+\operatorname{Hom}_{R}(P, P) \otimes_{\mathbf{Z}} \tilde{\mathbf{Z}}\left[\mathcal{X}_{+}\right]\right)$was shown to satisfy the induction hypothesis. We have an extension of groups

$$
\begin{aligned}
&\left(\mathbf{1}_{P}+\operatorname{Hom}_{\mathcal{M}_{R}}(P, P) \otimes_{\mathbf{Z}} \tilde{\mathbf{Z}}\left[\mathcal{X}_{+}^{n}\right]\right) \\
& \rightarrow\left(\mathbf{1}_{P}+\operatorname{Hom}_{\mathcal{M}_{R}}(P, P) \otimes_{\mathbf{Z}} \tilde{\mathbf{Z}}\left[\mathcal{X}_{+} \vee \mathcal{X}_{+}^{2} \vee \cdots \vee \mathcal{X}_{+}^{n}\right]\right) \\
& \quad \stackrel{\pi}{\rightarrow}\left(\mathbf{1}_{P}+\operatorname{Hom}_{\mathcal{M}_{R}}(P, P) \otimes_{\mathbf{Z}} \tilde{\mathbf{Z}}\left[\mathcal{X}_{+} \vee \mathcal{X}_{+}^{2} \vee \cdots \vee \mathcal{X}_{+}^{(n-1)}\right]\right) .
\end{aligned}
$$

Taking the bar construction we obtain a Kan fibration of cubes. By induction, the cube in the base satisfies the induction hypothesis, and so if the cube in the fiber does also, then since homotopy pullbacks commute (and these are cubes of connected spaces) the induction hypothesis will hold for the extension cube. The cube $B .\left(\mathbf{1}_{P}+\right.$ $\left.\operatorname{Hom}_{\mathcal{M}_{R}}(P, P) \otimes_{\mathbf{Z}} \tilde{\mathbf{Z}}\left[\mathcal{X}_{+}^{n}\right]\right)$ satisfies

$$
\begin{aligned}
& B .\left(\mathbf{1}_{P}+\operatorname{Hom}_{\mathcal{M}_{R}}(P, P) \otimes_{\mathbf{z}} \tilde{\mathbf{Z}}\left[\mathcal{X}_{+}^{n}\right]\right) \cong B .\left(\operatorname{Hom}_{\mathcal{M}_{R}}(P, P) \otimes_{\mathbf{z}} \tilde{\mathbf{Z}}\left[\mathcal{X}_{+}^{n}\right]\right) \\
& \cong \operatorname{Hom}_{\mathcal{M}_{R}}(P, P) \otimes_{\mathbf{Z}} B .\left(\tilde{\mathbf{Z}}\left[\mathcal{X}_{+}^{n}\right]\right) \cong \operatorname{Hom}_{\mathcal{M}_{R}}(P, P) \otimes_{\mathbf{Z}} \tilde{\mathbf{Z}}\left[(\Sigma \mathcal{X})_{+}^{n}\right] .
\end{aligned}
$$

Since $\Sigma \mathcal{X}$ is again a strongly co-Cartesian $S$-cube with the maps $\Sigma \mathcal{X}(\emptyset) \rightarrow \Sigma \mathcal{X}(s)$ $k_{s}+1$ connected for all $s \in S$, for all $T \neq \emptyset, \partial_{S-T} \Sigma \mathcal{X}$ is a $T$-strongly co-Cartesian cube with $k_{t}+1$ connectivity for all $t \in T$ and so the induction hypothesis is satisfied by example 4.4 of [G2] (for the functor $X \mapsto \tilde{\mathbf{Z}}\left[X_{+}^{n}\right]$ ). 


\section{The Main Theorem and its Corollaries}

Theorem 3.1 For $R$ a unital ring, for every $n$ there is a natural transformation of functors of simplicial R-bimodules

$$
\Phi_{n}: \Sigma W_{n}(R ;) \stackrel{\simeq}{\rightarrow} \mathbf{P}_{n} \tilde{K}\left(\mathcal{T}_{R}^{\pi}()\right)
$$

such that $\Phi_{n-1} \circ \Sigma \mathrm{res}_{n} \simeq p_{n} \circ \Phi_{n}$, which is a homotopy equivalence at any simplicial $R$-bimodule.

Note that for $M$ which are flat on one side, making the tensoring down map $M^{\otimes_{\hat{R}} n} \rightarrow M^{\otimes_{R} n}$ into a weak equivalence for every $n$, we get that $\mathcal{T}_{R}(M) \simeq$ $T_{R}(M)$, so $\mathcal{T}_{R}(M) / I^{n+1} \simeq T_{R}(M) / I^{n+1}$ and $\mathcal{T}_{R}^{\pi}(M) \simeq T_{R}^{\pi}(M)$. Therefore by [W2], $\tilde{K}\left(\mathcal{T}_{R}(M)\right) \simeq \tilde{K}\left(T_{R}(M)\right)$ and $\tilde{K}\left(\mathcal{T}_{R}^{\pi}(M)\right) \simeq \tilde{K}\left(T_{R}^{\pi}(M)\right)$.

For the definition and properties of the $W_{n}$, see [LMcC1]. It is the inverse limit over all $i \leq n$ of the $C_{i}$ fixedpoints in the cyclic derived tensor over $R$ of $i$ copies

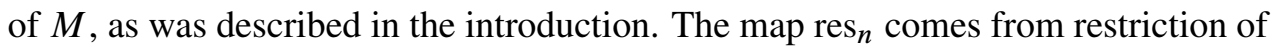
categories over which limits are taken from $\{1,2, \ldots, n\}$ to $\{1,2, \ldots, n-1\} ; p_{n}$ are the connecting maps of the Goodwillie tower of the functor.

Theorem 9.2 in [LMcC1] shows that for a connected pointed simplicial set $X$ and a simplicial $R$-bimodule $M, \tilde{K}(R ; \tilde{M}[X]) \stackrel{\simeq}{\rightarrow} W(R ; \tilde{M}[X])$. Corollary 9.3 there shows that the finite stages of Goodwillie's Taylor tower of the functor $K(R ; \tilde{M}[])$ from pointed simplicial sets to spectra are given by $\mathbf{P}_{n} \tilde{K}(R ; \tilde{M}[])=W_{n}(R ; \tilde{M}[])$. From this, one can deduce that for connected $R$-bimodules $M$,

$$
\tilde{K}(R ; M) \stackrel{\simeq}{\rightarrow} W(R ; M)
$$

and that Goodwillie's Taylor tower of the functor $K(R ; \quad)$ from simplicial $R$ bimodules to spectra is

$$
\mathbf{P}_{n} \tilde{K}(R ;)=W_{n}(R ;) .
$$

To get (3.1) from Theorem 9.2 of [LMcC1], observe that the functors on both sides of (3.1) commute with realizations for connected $R$-bimodules: $\tilde{K}(R ;) \simeq$ $\tilde{K}(R \ltimes \Sigma)$ commutes with realizations by [W2] for any $R$-bimodules; the finite stages of the Taylor tower $W_{n}(R ; \quad)$ commute with realizations as finite inverse limits of the $U_{a}(R ; \quad)^{C_{a}}$ which are directly seen to commute with realizations, but for $M$ connected the map $W(R ; M) \rightarrow W_{n}(R ; M)$ is $n$-connected, that is, it can be as connected as we like by taking $n$ large enough, and therefore $W(R ;$ ) commutes with realizations for connected bimodules. So we can take a connected $R$-bimodule $M$, and replace it by a homotopy equivalent realization of a bisimplicial 
set, assigning to each $n$ a simplicial set of the form covered by Theorem 9.2 in [LMcC1]: Given a general connected simplicial $R$-bimodule $M$, we can first represent it by a reduced one (that has only a single 0 -simplex, the basepoint) by looking at the sub-simplicial bimodule $M_{0}$ consisting of all the simplices in $M$ all of whose vertices are at the basepoint. The inclusion $M_{0} \hookrightarrow M$ is an equivalence on $\pi_{0}$ by assumption, and on all higher homotopy groups by the definition of the homotopy groups of a simplicial abelian group. Then, replace $M_{0}$ by its $R \otimes R^{\text {op }}$ free simplicial resolution

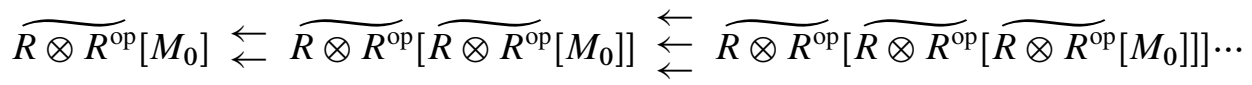

in which each stage is of the form $\tilde{N}[X]$ for $N=R \otimes R^{\mathrm{op}}$ an $R$-bimodule and $X$ a connected simplicial set.

To determine Goodwillie's Taylor tower as in (3.2), it suffices to look at the functor on connected bimodules, where we have (3.1). Having (3.1) and knowing from Corollary 5.9 of $[\mathrm{LMcC} 1]$ that

$$
U^{n}(R ; M)_{h C_{n}} \simeq \operatorname{hofib}\left(W_{n}(R ; M) \stackrel{\text { res }_{n}}{\rightarrow} W_{n-1}(R ; M)\right),
$$

that is, that to get from $W_{n-1}$ to $W_{n}$ one adds a homogenous degree $n$ functor, gives us an inductive proof of (3.2).

It follows from (3.2) above that $\Sigma W_{n}(R ;)=\mathbf{P}_{n}(\Sigma \tilde{K}(R ;))$. What Theorem 3.1 in fact shows is that there exists a natural transformation inducing an equivalence between Goodwillie Taylor towers of the functors $\Sigma \tilde{K}(R ; \quad)$ and $\tilde{K}\left(\mathcal{T}_{R}^{\pi}(\quad)\right)$. Moreover, we can draw the following

Corollary 3.2 For any unital ring $R$, there is a natural equivalence of functors of simplicial R-bimodules

$$
\Sigma W(R ;) \rightarrow \operatorname{holim}_{n} \tilde{K}\left(\mathcal{T}_{R}() / I^{n+1}\right) .
$$

Proof: (Of Corollary 3.2, given Theorem 3.1) We have, by Theorem 3.1 above, that for any $R$-bimodule $M$,

$$
\begin{aligned}
\Sigma W(R ; M) \stackrel{\text { def }}{=} \operatorname{holim}_{k} \Sigma W_{k}(R ; M) \simeq & \operatorname{holim}_{k} \mathbf{P}_{k} \tilde{K}\left(\mathcal{T}_{R}^{\pi}(M)\right) \\
& \stackrel{\text { def }}{=} \operatorname{holim}_{k} \mathbf{P}_{k} \tilde{K}\left(\operatorname{holim}_{n} \mathcal{T}_{R}(M) / I^{n+1}\right)
\end{aligned}
$$

But the Taylor tower of the functor $K\left(\operatorname{holim}_{n} \mathcal{T}_{R}() / I^{n+1}\right)$ can be determined by applying it to $M$ connected, where the map $\operatorname{holim}_{n} \mathcal{T}_{R}(M) / I^{n+1} \rightarrow \mathcal{T}_{R}(M) / I^{n_{0}+1}$ can be as connected as we want it to be, and $\tilde{K}$ preserves connectivity of maps, so

$$
\mathbf{P}_{k} \tilde{K}\left(\operatorname{holim}_{n} \mathcal{T}_{R}(M) / I^{n+1}\right)=\operatorname{holim}_{n} \mathbf{P}_{k} \tilde{K}\left(\mathcal{T}_{R}(M) / I^{n+1}\right),
$$


which we can plug into equation (3.3) to get

$$
\begin{aligned}
\Sigma W(R ; M) & \simeq \operatorname{holim}_{k} \operatorname{holim}_{n} \mathbf{P}_{k} \tilde{K}\left(\mathcal{T}_{R}(M) / I^{n+1}\right) \\
& \simeq \operatorname{holim}_{n} \operatorname{holim}_{k} \mathbf{P}_{k} \tilde{K}\left(\mathcal{T}_{R}(M) / I^{n+1}\right) \simeq \operatorname{holim}_{n} \tilde{K}\left(\mathcal{T}_{R}(M) / I^{n+1}\right),
\end{aligned}
$$

where the last equality is the convergence of the Taylor tower for $\tilde{K}\left(\mathcal{T}_{R}() / I^{n+1}\right)$ from Proposition 2.1 above.

Corollary 3.3 If $R$ is a unital ring, there is a natural equivalence of functors of connected simplicial R-bimodules

$$
\Phi: \Sigma \tilde{K}(R ;) \rightarrow \tilde{K}\left(\mathcal{T}_{R}()\right) .
$$

Proof: (Of Corollary 3.3, given Theorem 3.1) The natural transformation $\Phi$ is that introduced in the beginning of the proof of Theorem 3.1, which induces the $\Phi_{n}$ 's. The point is that for connected $M$, both the Taylor tower of $\Sigma \tilde{K}(R ;)$ converges to $\Sigma \tilde{K}(R ; M)$ (since that of $\tilde{K}(R ;)$ converges to $\tilde{K}(R ; M)$ by (3.1) and (3.2) above), and the Taylor tower of $\tilde{K}\left(\mathcal{T}_{R}^{\pi}()\right)$ converges to $\tilde{K}\left(\mathcal{T}_{R}^{\pi}(M)\right)$ : by Proposition 2.1 above, the Taylor towers converge for $\tilde{K}\left(\mathcal{T}_{R}(M) / I^{n+1}\right)$, that is $\tilde{K}\left(\mathcal{T}_{R}(M) / I^{n+1}\right) \simeq$ $\operatorname{holim}_{k} \mathbf{P}_{k} \tilde{K}\left(\mathcal{T}_{R}(M) / I^{n+1}\right)$. The map $\mathcal{T}_{R}^{\pi}(M) \rightarrow \mathcal{T}_{R}(M) / I^{n+1}$ is as connected as we want it to be for $n$ large enough, and since $\tilde{K}$ ( ) preserves connectivity of maps by [W2], we get that

$$
\begin{aligned}
\tilde{K}\left(\mathcal{T}_{R}^{\pi}(M)\right) \simeq \operatorname{holim}_{n} \tilde{K}\left(\mathcal{T}_{R}(M) / I^{n+1}\right) \simeq \operatorname{holim}_{n} \operatorname{holim}_{k} \mathbf{P}_{k} \tilde{K}\left(\mathcal{T}_{R}(M) / I^{n+1}\right) \\
\simeq \operatorname{holim}_{k} \operatorname{holim}_{n} \mathbf{P}_{k} \tilde{K}\left(\mathcal{T}_{R}(M) / I^{n+1}\right) \simeq \operatorname{holim}_{k} \mathbf{P}_{k} \tilde{K}\left(\mathcal{T}_{R}^{\pi}(M)\right)
\end{aligned}
$$

This shows that $\Phi: \Sigma \tilde{K}(R ;) \stackrel{\simeq}{\rightarrow} \tilde{K}\left(\mathcal{T}_{R}^{\pi}()\right)$ for $M$ connected, but of course for such $M$, the map

$$
\mathcal{T}_{R}(M) \rightarrow \mathcal{T}_{R}^{\pi}(M)
$$

is an equivalence.

In fact, since the map $\mathcal{T}_{R}(M) \rightarrow \mathcal{T}_{R}^{\pi}(M)$ is an equivalence for connected $M$, it is of order $n$ for all $n$ and hence $\mathbf{P}_{n} K\left(\mathcal{T}_{R}()\right) \stackrel{\sim}{\rightarrow} \mathbf{P}_{n} K\left(\mathcal{T}_{R}^{\pi}()\right)$ for all $n$, so the convergence of the Taylor tower for $\tilde{K}^{\pi}\left(\mathcal{T}_{R}()\right)$ for connected $M$ is in fact the convergence of the Taylor tower for $\tilde{K}\left(\mathcal{T}_{R}()\right)$ for such $M$ as well. 
Proof: (Of Theorem 3.1) The augmentation ideal $I$ for $\mathcal{T}_{R}^{\pi}(M) \rightarrow R$ for any $M$ is such that $1+I$ is contained in the units of $\mathcal{T}_{R}^{\pi}(M)$ and hence the fiber of the map $K\left(\mathcal{T}_{R}^{\pi}(M)\right) \rightarrow K(R)$ can by section I.2.5 of [DGMcC] be modeled as the stabilization in Waldhausen's S-construction of the functor

$$
\bigvee_{P \in \mathcal{P}_{R}} B .\left(\mathbf{1}_{P}+I_{P}\left(\mathcal{T}_{R}^{\pi}(M)\right)\right)
$$

As before, $I_{P}\left(\mathcal{T}_{R}^{\pi}(M)\right)=\operatorname{Hom}_{\mathcal{M}_{R}}(P, P \otimes I)$ is considered as the ideal given by the kernel of the ring map $\operatorname{Hom}_{\mathcal{M}_{R}}\left(P, P \otimes_{R} \mathcal{T}_{R}^{\pi}(M)\right) \rightarrow \operatorname{Hom}_{\mathcal{M}_{R}}(P, P)$.

We define a natural transformation

$$
\Phi: \Sigma \tilde{K}(R ; M) \rightarrow \tilde{K}\left(\mathcal{T}_{R}^{\pi}(M)\right)
$$

as the stablization of the natural transformation between the model of $\tilde{K}(R ; M)$ as the stabilization of $\bigvee_{P \in \mathcal{P}_{R}} \operatorname{Hom}_{\mathcal{M}_{R}}\left(P, P \otimes_{R} M\right)$ and the above model of $\tilde{K}\left(\mathcal{T}_{R}^{\pi}(M)\right)$ which for a map $m \in \operatorname{Hom}_{\mathcal{M}_{R}}\left(P, P \otimes_{R} M\right)$ sends

$$
m \mapsto(1-m)^{-1}=\Sigma_{i=0}^{\infty} m^{\otimes_{R} i} .
$$

The point is that 0 -simplices in $\tilde{K}(R ; M)$ become 1 -simplices in its suspension; each such 1 -simplex which comes from the 0 -simplex $m$ is mapped to a 1 -simplex in the classifying space corresponding to the element

$$
\Sigma_{i=0}^{\infty} m^{\otimes_{R} i} \in B_{1}\left(\mathbf{1}_{P}+I_{P}\left(\mathcal{T}_{R}^{\pi}(M)\right)\right)=\mathbf{1}_{P}+I_{P}\left(\mathcal{T}_{R}^{\pi}(M)\right) .
$$

Note that, for example, the notation $m^{\otimes_{R} 2}$ means the composition

$$
P \stackrel{m}{\rightarrow} P \otimes_{R} M \stackrel{m \otimes 1_{M}}{\longrightarrow} P \otimes_{R} M \otimes_{R} M
$$

and is therefore also in $I_{P}\left(\mathcal{T}_{R}^{\pi}\right)$.

What we want to show is that this natural transformation $\Phi$ induces an equivalence of Goodwillie's Taylor towers at the basepoint $*$, and these are determined by what they do on sufficiently connected spaces. Thus, the theorem will follow once we show that $\Phi$ induces an equivalence after one suspension. We would like to establish the result using analytic continuation as in [G3]. In order to do this we first must observe that $\tilde{K}\left(\mathcal{T}_{R}^{\pi}(B).\right)$ commutes with realizations, has the limit axiom and is -1-analytic. These are all true because the fact that $\mathcal{T}_{R}^{\pi}(B.) \rightarrow \mathcal{T}_{R}(B.) / I^{n+1}$ is $n$ connected for all $n$ implies $\tilde{K}\left(\mathcal{T}_{R}^{\pi}(B).\right) \rightarrow \tilde{K}\left(\mathcal{T}_{R}(B.) / I^{n+1}\right)$ is $n$ connected for all $n$ and these results hold for $K\left(\mathcal{T}_{R}(B.) / I^{n+1}\right)$ for all $n$ by Proposition 2.1 above. 
Thus, we fix our $M$, which we may assume to be connected, and are interested in the fibers of $\Sigma \tilde{K}(R ; M \oplus N) \rightarrow \Sigma \tilde{K}(R ; M)$ and of $\tilde{K}\left(\mathcal{T}_{R}^{\pi}(M \oplus N)\right) \rightarrow \tilde{K}\left(\mathcal{T}_{R}^{\pi}(M)\right)$ in a $2 n$ range when $N$ is $n$-connected. Since we assume that $M$ is connected, $\mathcal{T}_{R}(M) \stackrel{\simeq}{\rightarrow} \mathcal{T}_{R}^{\pi}(M)$ and $\mathcal{T}_{R}(M \oplus N) \stackrel{\simeq}{\rightarrow} \mathcal{T}_{R}^{\pi}(M \oplus N)$.

For the fiber of $\Sigma \tilde{K}(R ; M \oplus N) \rightarrow \Sigma \tilde{K}(R ; M)$, we can describe it using [LMcC1] which shows that for connected bimodules $\tilde{K}(R,) \simeq W(R ;)$ (that is, for connected bimodules the Taylor tower converges to $\tilde{K}(R ;))$ together with the splitting of Theorem 2.2 in [LMcC2] for $W(R$; ). We get that for $M, N$ connected,

$\tilde{K}(R ; M \oplus N)$

$$
=\bigvee_{a=1}^{\infty} \bigvee_{\{f:\{1, \ldots, a\} \rightarrow\{M, N\} \text { non periodic }\} / C_{a}} \tilde{K}\left(R ; f(1) \otimes_{R}^{\wedge} \cdots \otimes_{R}^{\wedge} f(a)\right),
$$

where $C_{a}$ acts on functions $\{1, \ldots, a\} \rightarrow\{M, N\}$ by permuting $\{1, \ldots, a\}$ cyclically before applying the function, and a function $f$ is considered periodic if for some $b \mid a$, the value of $f(i)$ is determined by the remainder of $i$ when divided by $b$, that is: when if we write the values of $f$ as a word of length $a$ in $M$ and $N$, that word is a word of length $b$ repeated $a / b$ times. It follows from the discussion there that the maps $M \hookrightarrow M \oplus N \stackrel{p_{1}}{\rightarrow} M$ embed $\tilde{K}(R ; M)$ as the direct summand corresponding to the function from the set of one element $1 \mapsto M$, so the homotopy fiber of the projection map consists of all the other summands.

The fiber of $\tilde{K}\left(\mathcal{T}_{R}(M \oplus N)\right) \rightarrow \tilde{K}\left(\mathcal{T}_{R}(M)\right)$ is exactly the algebraic K-theory of $\mathcal{T}_{R}(M \oplus N)$ reduced over $\mathcal{T}_{R}(M)$. We can compare this reduced algebraic K-theory to that of another ring: Note that, by sending any terms with more than one tensored entry in $N$ to the basepoint, we have a $2 n$-connected multiplicative map

$$
\mathcal{T}_{R}(M \oplus N) \stackrel{\Psi}{\rightarrow} \mathcal{T}_{R}(M) \ltimes\left(\mathcal{T}_{R}(M) \otimes_{R}^{\wedge} N \otimes_{R}^{\wedge} \mathcal{T}_{R}(M)\right) .
$$

We can put all this together in a commutative diagram

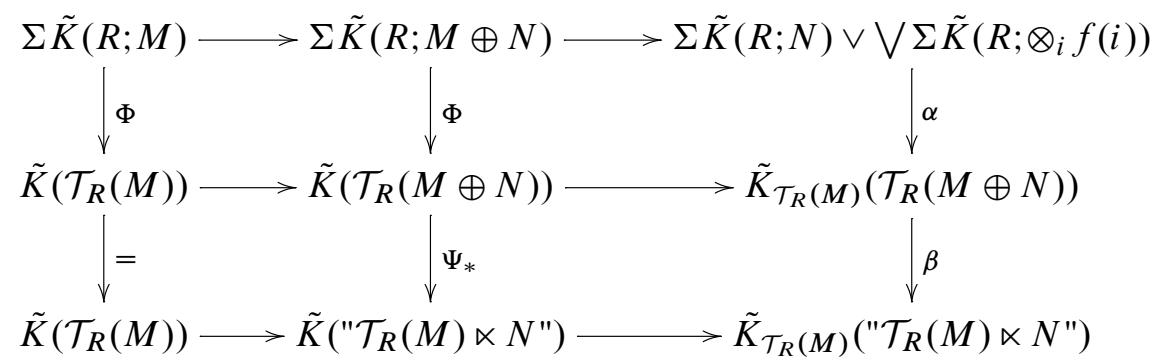


where

$$
\begin{aligned}
\bigvee \Sigma \tilde{K}(R ; & \left.\otimes_{i} f(i)\right) \\
& =\bigvee_{a=2}^{\infty}\{f:\{1, \ldots, a\} \rightarrow\{M, N\} \text { non periodic }\} / C_{a} \\
& \Sigma \tilde{K}\left(R ; f(1) \otimes_{R}^{\wedge} \cdots \otimes_{R}^{\wedge} f(a)\right)
\end{aligned}
$$

and

$$
" \mathcal{T}_{R}(M) \ltimes N "=\mathcal{T}_{R}(M) \ltimes\left(\mathcal{T}_{R}(M) \otimes_{R}^{\wedge} N \otimes_{R} \mathcal{T}_{R}(M)\right) .
$$

The left column maps to the center column by maps induced by the obvious inclusions. Since the inclusions are all inclusions of retracts, the spectra in the center column all split as the product of the spectrum on their left and the spectrum on their right.

Our goal is to show that when $N$ is $n$-connected, $\alpha$ is $2 n$-connected in equation (3.5). This would mean that $\Phi$ induces an equivalence of the Goodwillie differentials at $M$. Since $\Psi$ is $2 n$-connected, $\Psi_{*}$ and therefore also $\beta$ are $2 n$ connected as well. So our strategy will be to show that $\beta \circ \alpha$ is $2 n$-connected, and deduce from that that $\alpha$ is.

It is plausible that $\beta \circ \alpha$ is $2 n$-connected, since we will now see that its target and source have the same homotopy type in these dimensions. In the next sections, we will see that $\beta \circ \alpha$ actually induces a $2 n$-equivalence.

We can map

$$
\begin{array}{r}
\Sigma \tilde{K}(R ; N) \vee \bigvee_{a=2}^{\infty} \bigvee_{\{f:\{1, \ldots, a\} \rightarrow\{M, N\} \text { non periodic }\} / C_{a}} \Sigma \tilde{K}\left(R ; f(1) \otimes_{R}^{\wedge} \cdots \otimes_{R}^{\wedge} f(a)\right) \\
\stackrel{2 n}{\rightarrow} \bigvee_{a=0}^{\infty} \Sigma \tilde{K}\left(R ; M^{\otimes_{R} a} \otimes_{R}^{\wedge} N\right) \stackrel{2 n}{\rightarrow} \bigvee_{a=0}^{\infty} \Sigma \tilde{T H H}\left(R ; M^{\otimes_{R} a} \otimes_{R}^{\wedge} N\right) \\
\simeq \operatorname{THH}\left(R ; \mathcal{T}_{R}(M) \otimes_{R}^{\wedge} \Sigma N\right)
\end{array}
$$

where the first map collapses all terms corresponding to $f$ 's which hit $N$ more than once, and since reduced K-theory sends $2 n$-connected bimodules to $2 n$-connected spectra, it is $2 n$-connected; the second map is $2 n$-connected by [DMcC1], and the last map is an equivalence by the linearity of THH in the bimodule variable.

By [DMcC1],

$$
\begin{aligned}
\tilde{K}_{\mathcal{T}_{R}(M)}\left(\mathcal{T}_{R}(M) \ltimes \mathcal{T}_{R}(M) \otimes_{R}^{\wedge} N\right. & \left.\otimes_{R}^{\wedge} \mathcal{T}_{R}(M)\right) \\
& \simeq K\left(\mathcal{T}_{R}(M) ; \Sigma \mathcal{T}_{R}(M) \otimes_{R}^{\wedge} N \otimes_{R}^{\wedge} \mathcal{T}_{R}(M)\right) \\
& \stackrel{2 n}{\rightarrow} \operatorname{THH}\left(\mathcal{T}_{R}(M) ; \Sigma \mathcal{T}_{R}(M) \otimes_{R}^{\wedge} N \otimes_{R}^{\wedge} \mathcal{T}_{R}(M)\right)
\end{aligned}
$$


and by Lemma 4.2 below, there is a homotopy equivalence

$\operatorname{THH}\left(R ; \mathcal{T}_{R}(M) \otimes_{R}^{\wedge} \Sigma N\right) \stackrel{\simeq}{\rightarrow} \operatorname{THH}\left(\mathcal{T}_{R}(M) ; \mathcal{T}_{R}(M) \otimes_{R}^{\wedge} \Sigma N \otimes_{R}^{\wedge} \mathcal{T}_{R}(M)\right)$, the same spectrum we ended up with in equation (3.6).

\section{Checking that the Equivalence is Induced by the Correct Map}

This section is dedicated to finishing the proof of Theorem 3.1 by tracing the maps in (3.5) to establish that $\beta \circ \alpha$ in fact induces a $2 n$-equivalence for $N n$-connected. We will first need some lemmas, which will all be proven in the last section of the paper.

Lemma 4.1 Model $\tilde{K}(R ; M \oplus N)$ by Waldhausen's S-construction as the stabilization of

$$
\bigvee_{P \in S^{(n)} \mathcal{P}_{R}} \operatorname{Hom}_{R}\left(P, P \otimes_{R}(M \oplus N)\right) \cong \bigvee_{P \in S^{(n)} \mathcal{P}_{R}}\left(\operatorname{Hom}_{R}(P, P \otimes M) \oplus \operatorname{Hom}_{R}(P, P \otimes N)\right) ;
$$

model, similarly,

$$
\begin{aligned}
\tilde{K}_{\mathcal{T}_{R}(M)}\left(\mathcal { T } _ { R } ( M ) \ltimes \left(\mathcal{T}_{R}(M) \otimes_{R}^{\wedge} N\right.\right. & \left.\left.\otimes_{R}^{\wedge} \mathcal{T}_{R}(M)\right)\right) \\
& \simeq \tilde{K}\left(\mathcal{T}_{R}(M) ; B .\left(\mathcal{T}_{R}(M) \otimes_{R}^{\wedge} N \otimes_{R}^{\wedge} \mathcal{T}_{R}(M)\right)\right)
\end{aligned}
$$

(this is the homotopy equivalence of [DMcC1]) as the stabilization of

$$
\bigvee_{Q \in S^{(n)} \mathcal{P}_{\mathcal{T}_{R}(M)}} \operatorname{Hom}_{\mathcal{T}_{R}(M)}\left(Q, Q \otimes_{\mathcal{T}_{R}(M)} B .\left(\mathcal{T}_{R}(M) \otimes_{R}^{\wedge} N \otimes_{R}^{\wedge} \mathcal{T}_{R}(M)\right)\right)
$$

Then if we start at the middle of the top row of diagram (3.5), follow the maps $\Phi$ and $\Psi_{*}$ down and then the map which goes right, the resulting map

$$
\begin{aligned}
\tilde{K}(R ; M \oplus N) \rightarrow \tilde{K}_{\mathcal{T}_{R}(M)}\left(\mathcal{T}_{R}(M)\right. & \left.\ltimes\left(\mathcal{T}_{R}(M) \otimes_{R}^{\wedge} N \otimes_{R}^{\wedge} \mathcal{T}_{R}(M)\right)\right) \\
& \simeq \tilde{K}\left(\mathcal{T}_{R}(M) ; B .\left(\mathcal{T}_{R}(M) \otimes_{R}^{\wedge} N \otimes_{R}^{\wedge} \mathcal{T}_{R}(M)\right)\right)
\end{aligned}
$$

is induced by sending the suspension $\Sigma(m, n)$ of each $(m, n) \in \operatorname{Hom}_{R}(P, P \otimes M) \oplus$ $\operatorname{Hom}_{R}(P, P \otimes N)$ to

$$
\begin{aligned}
& \left(\mathbf{1}_{P \otimes_{R} \mathcal{T}_{R}(M)}-m\right)^{-1} \otimes n \\
& \in \operatorname{Hom}_{\mathcal{T}_{R}(M)}\left(P \otimes_{R} \mathcal{T}_{R}(M), P \otimes_{R} \mathcal{T}_{R}(M) \otimes_{\mathcal{T}_{R}(M)} \mathcal{T}_{R}(M) \otimes_{R}^{\wedge} N \otimes_{R}^{\wedge} \mathcal{T}_{R}(M)\right) \\
= & \operatorname{Hom}_{\mathcal{T}_{R}(M)}\left(P \otimes_{R} \mathcal{T}_{R}(M), P \otimes_{R} \mathcal{T}_{R}(M) \otimes_{\mathcal{T}_{R}(M)} B_{1}\left(\mathcal{T}_{R}(M) \otimes_{R}^{\wedge} N \otimes_{R}^{\wedge} \mathcal{T}_{R}(M)\right)\right)
\end{aligned}
$$

in the summand corresponding to $Q=P \otimes_{R} \mathcal{T}_{R}(M)$. 
Lemma 4.2 Let $R$ be a ring spectrum, and $S$ an $R$-algebra. Let $X$ be an $S-R$ bimodule. Then there is a homotopy equivalence

$$
\operatorname{THH}(R ; X) \simeq \operatorname{THH}\left(S ; X \otimes_{R}^{\wedge} S\right) .
$$

Lemma 4.3 Let $R$ be a simplicial ring, $S$ a simplicial $R$-algebra, and $X$ a simplicial $S-R$ bimodule. Then if we construct $\mathrm{THH}(R ; M)$ for an $R$-bimodule $M$ via the Waldhausen $S$-construction

$$
\left\{n \mapsto \oplus_{P \in S^{(n)} \mathcal{P}_{R}} \operatorname{Hom}_{S^{(n)} \mathcal{M}_{R}}\left(P, P \otimes_{R} M\right)\right\},
$$

the isomorphism

$$
\operatorname{THH}(R ; X) \simeq \operatorname{THH}\left(R ; S \otimes_{R}^{\wedge} X\right)
$$

of Lemma 4.2 for the associated spectra is induced by the map

$\oplus_{P \in S^{(n)} \mathcal{P}_{R}} \operatorname{Hom}_{S^{(n)} \mathcal{M}_{R}}\left(P, P \otimes_{R} X\right) \rightarrow \oplus_{Q \in S^{(n)} \mathcal{P}_{S}} \operatorname{Hom}_{S^{(n)} \mathcal{M}_{S}}\left(Q, Q \otimes_{S}\left(X \otimes_{R}^{\wedge} S\right)\right)$ sending

$$
\alpha: P \rightarrow P \otimes_{R} X
$$

to

$$
\alpha \otimes_{R}^{\wedge} \mathbf{1}_{S}: P \otimes_{R}^{\wedge} S=P \otimes_{R} S \rightarrow P \otimes_{R} S \otimes_{S} X \otimes_{R}^{\wedge} S \simeq P \otimes_{R} X \otimes_{R}^{\wedge} S .
$$

Using these lemmas, we will be able to complete our proof of Theorem 3.1. The [DMcC1] map

$$
\begin{aligned}
\tilde{K}\left(\mathcal{T}_{R}(M) ; B .\left(\mathcal{T}_{R}(M) \otimes_{R}^{\wedge}\right.\right. & \left.\left.N \otimes_{R}^{\wedge} \mathcal{T}_{R}(M)\right)\right) \\
& \stackrel{2 n}{\rightarrow} \operatorname{THH}\left(\mathcal{T}_{R}(M) ; B .\left(\mathcal{T}_{R}(M) \otimes_{R}^{\wedge} N \otimes_{R}^{\wedge} \mathcal{T}_{R}(M)\right)\right)
\end{aligned}
$$

is obtained simply by passing from $\bigvee$ to $\bigoplus$,

$$
\begin{aligned}
& \bigvee_{Q \in \mathcal{R}_{\mathcal{T}_{R}(M)}} \operatorname{Hom}_{\mathcal{T}_{R}(M)}\left(Q, Q \otimes_{\mathcal{T}_{R}(M)} B .\left(\mathcal{T}_{R}(M) \otimes_{R}^{\wedge} N \otimes_{R}^{\wedge} \mathcal{T}_{R}(M)\right)\right) \\
& \quad \rightarrow \bigoplus_{Q \in \mathcal{R}_{\mathcal{T}^{(}(M)}} \operatorname{Hom}_{\mathcal{T}_{R}(M)}\left(Q, Q \otimes_{\mathcal{T}_{R}(M)} B .\left(\mathcal{T}_{R}(M) \otimes_{R}^{\wedge} N \otimes_{R}^{\wedge} \mathcal{T}_{R}(M)\right)\right) .
\end{aligned}
$$

So by Lemma 4.1 above, the composition of $\Psi_{*} \circ \Phi$ with the [DMcC1] linearization map (4.1) sends the loop represented by the 1 -simplex $\Sigma(m, n)$ in the $P$ summand 
to something homotopic to the loop represented by the 1-simplex $\left(\mathbf{1}_{P \otimes_{R} \mathcal{T}_{R}(M)}-\right.$ $m)^{-1} \otimes n$ in the $P \otimes_{R} \mathcal{T}_{R}(M)$ summand.

Alternatively, if we look at the map onto the cofiber on the top row of equation (3.5) and then collapse to a point the terms with more than one $N$,

$$
\begin{aligned}
& \tilde{K}(R ; M \oplus N) \\
& \rightarrow \tilde{K}(R: N) \vee \bigvee_{a=2}^{\infty} \bigvee_{\{f:\{1, \ldots, a\} \rightarrow\{M, N\} \text { non periodic }\} / C_{a}} \tilde{K}\left(R ; f(1) \otimes_{R}^{\wedge} \cdots \otimes_{R}^{\wedge} f(a)\right) \\
& \stackrel{2 n}{\rightarrow} \bigvee_{a=0}^{\infty} \tilde{K}\left(R ; M^{\otimes_{\hat{R}} a} \otimes_{R}^{\wedge} N\right) \simeq \prod_{a=0}^{\infty} \tilde{K}\left(R ; M^{\otimes_{R} a} \otimes_{R}^{\wedge} N\right)
\end{aligned}
$$

it is induced by the stabilization of the product of the maps

$$
\bigvee_{P \in \mathcal{P}_{R}}\left(\operatorname{Hom}_{R}(P, P \otimes M) \oplus \operatorname{Hom}_{R}(P, P \otimes N)\right) \rightarrow \bigvee_{P \in \mathcal{P}_{R}} \operatorname{Hom}_{R}\left(P, P \otimes M^{\otimes} \hat{R}^{a} \otimes \hat{R}^{\wedge} N\right)
$$

sending

$$
(m, n) \mapsto m^{\otimes a} \otimes n .
$$

To see this, we use the fact that $M$ is connected and $N$ is $n$-connected for $n$ which we may assume to be at least 1 , and therefore $M \oplus N$ is connected as well. Then

$$
\begin{aligned}
& \tilde{K}(R ; M \oplus N) \stackrel{\simeq}{\rightarrow} W(R ; M \oplus N) \\
& \simeq \prod_{a=1}^{\infty} \bigvee_{\{f:\{1, \ldots, a\} \rightarrow\{M, N\} \text { non periodic }\} / C_{a}} W\left(R ; f(1) \otimes_{R}^{\wedge} \cdots \otimes_{R}^{\wedge} f(a)\right) \\
& \stackrel{2 n}{\rightarrow} W(R ; M) \times \prod_{a=0}^{\infty} W\left(R ; M^{\otimes_{\hat{R}} a} \otimes_{R}^{\wedge} N\right) \stackrel{\simeq}{\leftarrow} \tilde{K}(R ; M) \times \prod_{a=0}^{\infty} \tilde{K}\left(R ; M^{\otimes_{\hat{R}} a} \otimes_{R}^{\wedge} N\right)
\end{aligned}
$$

Here the first and last equivalences are by Theorem 9.2 in [ $\mathrm{LMcC} 1]$, the second one is the splitting of Theorem 2.2 in [LMcC2], and the third map is $2 n$-connected because if $f$ hits $N$ more than once, $f(1) \otimes_{R}^{\wedge} \cdots \otimes_{R}^{\wedge} f(a)$ and therefore also $W$ of $R$ with coefficients in it are $2 n$-connected.

We are, of course, quotienting this whole picture out by $\tilde{K}(R ; M)$. By following the decomposition of Theorem 2.2 in [ $\mathrm{LMcC} 2]$ on the 0 -dimensional part (as we did before), we see that

$$
(m, n) \in \operatorname{Hom}_{R}(P, P \otimes M) \oplus \operatorname{Hom}_{R}(P, P \otimes N)
$$


in $\tilde{K}(R ; M \oplus N)$ in the beginning of equation (4.3) lands in the same place in $W(R ; M) \times \prod_{a=0}^{\infty} W\left(R ; M^{\otimes} \hat{R}_{R} a \otimes_{R}^{\wedge} N\right)$ as $\{m\} \times \prod_{a=0}^{\infty}\left\{m^{\otimes a} \otimes n\right\}$ in $\tilde{K}(R ; M) \times$ $\prod_{a=0}^{\infty} \tilde{K}\left(R ; M^{\otimes_{R}} a \otimes_{R}^{\wedge} N\right)$ on the right. Since the spectra we are looking at increase in connectivity, we know that their sum $\bigvee$ is homotopy equivalent to their product.

In (3.5), we are using the suspension of the map of (4.2),

$$
\Sigma \tilde{K}(R ; M \oplus N) \rightarrow \Sigma \bigvee_{a=0}^{\infty} \tilde{K}\left(R ; M^{\otimes_{R} a} \otimes_{R}^{\wedge} N\right)
$$

and want to compose it with

$$
\begin{aligned}
\Sigma \bigvee_{a=0}^{\infty} \tilde{K}\left(R ; M^{\otimes_{R} a}\right. & \left.\otimes_{R}^{\wedge} N\right) \stackrel{2 n+1}{\longrightarrow} \Sigma \bigvee_{a=0}^{\infty} \operatorname{THH}\left(R ; M^{\otimes_{R} a} \otimes_{R}^{\wedge} N\right) \\
& \simeq \operatorname{THH}\left(R ; \Sigma\left(\bigoplus_{a=0}^{\infty} M^{\otimes_{R} a} \otimes_{R}^{\wedge} N\right) \simeq \operatorname{THH}\left(R ; \Sigma \mathcal{T}_{R}(M) \otimes_{R}^{\wedge} N\right),\right.
\end{aligned}
$$

where the first map is that of [DMcC1], and the second uses the linearity of THH in the bimodule coordinate.

So the suspension of $(m, n) \in \operatorname{Hom}_{R}(P, P \otimes M) \oplus \operatorname{Hom}_{R}(P, P \otimes N)$ lands in the 1-simplex corresponding to

$$
\sum_{a=0}^{\infty} m^{\otimes a} \otimes n=\left(\mathbf{1}_{P}-m\right)^{-1} \otimes n \in \operatorname{Hom}_{R}\left(P, P \otimes_{R} \mathcal{T}_{R}(M) \otimes_{R}^{\wedge} N\right) .
$$

When we went the $\Psi_{*} \circ \Phi$ route, instead of getting

$$
\left(\mathbf{1}_{P}-m\right)^{-1} \otimes n \in \operatorname{Hom}_{R}\left(P, P \otimes_{R} \mathcal{T}_{R}(M) \otimes_{R}^{\wedge} N\right) \subset \operatorname{THH}\left(R ; \mathcal{T}_{R}(M) \otimes_{R}^{\wedge} N\right)
$$

we got

$$
\begin{aligned}
& \left(\mathbf{1}_{P \otimes_{R} \mathcal{T}_{R}(M)}-m\right)^{-1} \otimes n \\
& \in \operatorname{Hom}_{\mathcal{T}_{R}(M)}\left(P \otimes_{R} \mathcal{T}_{R}(M), P \otimes_{R} \mathcal{T}_{R}(M) \otimes_{\mathcal{T}_{R}(M)} \mathcal{T}_{R}(M) \otimes_{R}^{\wedge} N \otimes_{R}^{\wedge} \mathcal{T}_{R}(M)\right) \\
& \subset \operatorname{THH}\left(\mathcal{T}_{R}(M) ; \mathcal{T}_{R}(M) \otimes_{R}^{\wedge} N \otimes_{R}^{\wedge} \mathcal{T}_{R}(M)\right) .
\end{aligned}
$$

But by Lemmas 4.2 and 4.3 above, that is exactly what we need to assure ourselves that up to homotopy, the map $\beta \circ \alpha$ is the $2 n$-equivalence we are after. 


\section{Proofs of the Technical Lemmas}

\section{Lemma 4.1}

Proof: The discussion will be done over $\mathcal{P}_{R}$, i.e. in the first stage of the Waldhausen S-construction, but can be carried over to $S^{(n)} \mathcal{P}_{R}$ for any $n$.

The map

$$
\Sigma \tilde{K}(R ; M \oplus N) \stackrel{\Phi}{\rightarrow} \tilde{K}\left(\mathcal{T}_{R}(M \oplus N)\right)
$$

was induced by stabilizing the map

$$
\begin{gathered}
\Sigma \bigvee_{P \in \mathcal{P}_{R}} \operatorname{Hom}_{R}\left(P, P \otimes \otimes_{R}(M \oplus N)\right) \longrightarrow \bigvee_{P \in \mathcal{P}_{R}} B \cdot\left(\mathbf{1}_{P}+I_{P}\left(\mathcal{T}_{R}(M \oplus N)\right)\right) \\
\downarrow \\
\downarrow \\
\bigvee_{P \in \mathcal{P}_{R}}\left(\operatorname{Hom}_{R}(P, P \otimes M) \oplus \operatorname{Hom}_{R}(P, P \otimes N)\right)
\end{gathered}
$$

sending $\Sigma(m, n)$ to the 1-simplex

$$
\mathbf{1}_{P}+(m+n)+(m+n)^{\otimes 2}+(m+n)^{\otimes 3}+\cdots \in B_{1}\left(\mathbf{1}_{P}+I_{P}\left(\mathcal{T}_{R}(M \oplus N)\right)\right) .
$$

Now $\Psi_{*}: \tilde{K}\left(\mathcal{T}_{R}(M \oplus N)\right) \rightarrow \tilde{K}\left(\mathcal{T}_{R}(M) \ltimes\left(\mathcal{T}_{R}(M) \otimes_{R}^{\wedge} N \otimes_{R}^{\wedge} \mathcal{T}_{R}(M)\right)\right)$ is induced by

$$
\begin{aligned}
\Psi_{*}: \bigvee_{P \in \mathcal{P}_{R}} B .\left(\mathbf{1}_{P}\right. & \left.+I_{P}\left(\mathcal{T}_{R}(M \oplus N)\right)\right) \\
& \rightarrow \bigvee_{P \in \mathcal{P}_{R}} B .\left(\mathbf{1}_{P}+I_{P}\left(\mathcal{T}_{R}(M) \ltimes\left(\mathcal{T}_{R}(M) \otimes_{R}^{\wedge} N \otimes_{R}^{\wedge} \mathcal{T}_{R}(M)\right)\right)\right)
\end{aligned}
$$

so the original 1-simplex $\Sigma(m, n)$ will be further sent to the 1-simplex

$$
\begin{aligned}
\mathbf{1}_{P}+\sum_{i=1}^{\infty} m^{\otimes i}+\sum_{j, k=0}^{\infty} m^{\otimes j} \otimes & n \otimes m^{\otimes k} \\
& =\left(\mathbf{1}_{P}-m\right)^{-1}+\left(\mathbf{1}_{P}-m\right)^{-1} \otimes n \otimes\left(\mathbf{1}_{P}-m\right)^{-1} .
\end{aligned}
$$

Until now, we have looked at K-theory of $R$ algebras reduced over $R$, which we can emphasize by writing $\tilde{K}_{R}()$. To get $\tilde{K}_{\mathcal{T}_{R}(M)}\left(\mathcal{T}_{R}(M) \ltimes\left(\mathcal{T}_{R}(M) \otimes_{R}{ }_{R} N \otimes_{R}^{\wedge}\right.\right.$ $\left.\mathcal{T}_{R}(M)\right)$ ), we look at the homotopy fiber of the map

$$
\tilde{K}_{R}\left(\mathcal{T}_{R}(M) \ltimes\left(\mathcal{T}_{R}(M) \otimes_{R}^{\wedge} N \otimes_{R}^{\wedge} \mathcal{T}_{R}(M)\right)\right) \rightarrow \tilde{K}_{R}\left(\mathcal{T}_{R}(M)\right) .
$$


Since $\tilde{K}_{R}\left(\mathcal{T}_{R}(M)\right)$ is a direct summand in $\tilde{K}_{R}\left(\mathcal{T}_{R}(M) \ltimes\left(\mathcal{T}_{R}(M) \otimes_{R} N \otimes_{R}{ }_{R} \mathcal{T}_{R}(M)\right)\right)$, so is the homotopy fiber of the map, and when we map $\tilde{K}_{R}\left(\mathcal{T}_{R}(M) \ltimes\left(\mathcal{T}_{R}(M) \otimes_{R}^{\wedge}\right.\right.$ $\left.N \otimes_{R}^{\wedge} \mathcal{T}_{R}(M)\right)$ ) down to the homotopy fiber, the image of $\tilde{K}_{R}\left(\mathcal{T}_{R}(M)\right)$ is identified to a point. So on the pre-stabilized version, $\bigvee_{P \in \mathcal{P}_{R}} B .\left(\mathbf{1}_{P}+I_{P}\left(\mathcal{T}_{R}(M) \ltimes\right.\right.$ $\left.\left(\mathcal{T}_{R}(M) \otimes_{R}^{\wedge} N \otimes_{R}^{\wedge} \mathcal{T}_{R}(M)\right)\right)$ ), we know that anything coming from $\tilde{K}_{R}\left(\mathcal{T}_{R}(M)\right)$, that is $\bigvee_{P \in \mathcal{P}_{R}} B .\left(\mathbf{1}_{P}+I_{P}\left(\mathcal{T}_{R}(M)\right)\right)$, has to be identified to a point in the homotopy fiber.

But note that if we have a subgroup $H \subset G$ and collapse the subspace $B H \subset$ $B G$ to a point, in the quotient space $B G / B H$, for any $h \in H, g \in G$ the 1-simplex corresponding to $h g$ is homotopic via the 2-simplex $(h, g)$ (one of whose edges has been collapsed to a point) to the 1-simplex corresponding to $g$.

Since

$$
\begin{aligned}
\tilde{K}_{R}\left(\mathcal{T}_{R}(M)\right. & \left.\ltimes\left(\mathcal{T}_{R}(M) \otimes_{R}^{\wedge} N \otimes_{R}^{\wedge} \mathcal{T}_{R}(M)\right)\right) \\
& \simeq \tilde{K}_{R}\left(\mathcal{T}_{R}(M)\right) \times \tilde{K}_{\mathcal{T}_{R}(M)}\left(\mathcal{T}_{R}(M) \ltimes\left(\mathcal{T}_{R}(M) \otimes_{R}^{\wedge} N \otimes_{R}^{\wedge} \mathcal{T}_{R}(M)\right)\right),
\end{aligned}
$$

when we pass from $\tilde{K}_{R}$ to $\tilde{K}_{\mathcal{T}_{R}(M)}$, we are identifying $\tilde{K}_{R}\left(\mathcal{T}_{R}(M)\right)$ to a point, and so at each level of the stabilization, the image of our original 1-simplex $\Sigma(m, n)$ will be homotopic (rel endpoints) to the image of the 1-simplex

$$
\mathbf{1}_{P}+\left(\mathbf{1}_{P}-m\right)^{-1} \otimes n \in B_{1}\left(\mathbf{1}_{P}+I_{P}\left(\mathcal{T}_{R}(M) \ltimes\left(\mathcal{T}_{R}(M) \otimes_{R}^{\wedge} N \otimes_{R}^{\wedge} \mathcal{T}_{R}(M)\right)\right)\right) .
$$

Now when $\tilde{K}_{\mathcal{T}_{R}(M)}\left(\mathcal{T}_{R}(M) \ltimes\left(\mathcal{T}_{R}(M) \otimes_{R}^{\wedge} N \otimes_{R}^{\wedge} \mathcal{T}_{R}(M)\right)\right.$ is represented as the stabilization of

$$
\bigvee_{Q \in \mathcal{P}_{\mathcal{T}_{R}(M)}} B .\left(\mathbf{1}_{Q}+I_{Q}\left(\mathcal{T}_{R}(M) \ltimes\left(\mathcal{T}_{R}(M) \otimes_{R}^{\wedge} N \otimes_{R}^{\wedge} \mathcal{T}_{R}(M)\right)\right)\right),
$$

(note that the augmentation ideal here refers now to augmentation over $\mathcal{T}_{R}(M)$ ), then $\mathbf{1}_{P}+\left(\mathbf{1}_{P}-m\right)^{-1} \otimes n$ should be viewed there not as an $R$-linear map

$$
P \rightarrow P \otimes_{R}\left(\mathcal{T}_{R}(M) \ltimes\left(\mathcal{T}_{R}(M) \otimes_{R}^{\wedge} N \otimes_{R}^{\wedge} \mathcal{T}_{R}(M)\right)\right)
$$

but as its $\mathcal{T}_{R}(M)$-linear extension to

$$
\begin{aligned}
P \otimes_{R} \mathcal{T}_{R}(M) \rightarrow( & \left.P \otimes_{R} \mathcal{T}_{R}(M)\right) \otimes_{\mathcal{T}_{R}(M)}\left(\mathcal{T}_{R}(M) \ltimes\left(\mathcal{T}_{R}(M) \otimes_{R} N \otimes_{R} \mathcal{T}_{R}(M)\right)\right) \\
& \cong P \otimes_{R}\left(\mathcal{T}_{R}(M) \ltimes\left(\mathcal{T}_{R}(M) \otimes_{R}^{\wedge} N \otimes_{R}^{\wedge} \mathcal{T}_{R}(M)\right)\right) .
\end{aligned}
$$

(Extending maps $\mathcal{T}_{R}(M)$-linearly gives an isomorphism

$$
\left.\operatorname{Hom}_{R}\left(P, P \otimes_{R} S\right) \leftrightarrow \operatorname{Hom}_{\mathcal{T}_{R}(M)}\left(P \otimes_{R} \mathcal{T}_{R}(M),\left(P \otimes_{R} \mathcal{T}_{R}(M)\right) \otimes_{\mathcal{T}_{R}(M)} S\right) .\right)
$$


So now our original simplex $\Sigma(m, n)$ maps to the 1-simplex

$$
\begin{aligned}
& \mathbf{1}_{P \otimes_{R} \mathcal{T}_{R}(M)}+\left(\mathbf{1}_{\left.P \otimes_{R} \mathcal{T}_{R}(M)-m\right)^{-1} \otimes n}\right. \\
& \quad \in B_{1}\left(\mathbf{1}_{P \otimes_{R} \mathcal{T}_{R}(M)}+I_{P \otimes_{R} \mathcal{T}_{R}(M)}\left(\mathcal{T}_{R}(M) \ltimes\left(\mathcal{T}_{R}(M) \otimes_{R}^{\wedge} N \otimes_{R}^{\wedge} \mathcal{T}_{R}(M)\right)\right)\right)
\end{aligned}
$$

in the $\bigvee_{Q \in \mathcal{R}_{\mathcal{T}_{R}(M)}} B .\left(\mathbf{1}_{Q}+I_{Q}\left(\mathcal{T}_{R}(M) \ltimes\left(\mathcal{T}_{R}(M) \otimes_{R}^{\wedge} N \otimes_{R}^{\wedge} \mathcal{T}_{R}(M)\right)\right)\right)$ model of $\tilde{K}_{\mathcal{T}_{R}(M)}\left(\mathcal{T}_{R}(M) \ltimes\left(\mathcal{T}_{R}(M) \otimes_{R}^{\wedge} N \otimes_{R}^{\wedge} \mathcal{T}_{R}(M)\right)\right.$ which admits a homotopy equivalence

$$
\begin{aligned}
\tilde{K}\left(\mathcal{T}_{R}(M) ; B .\left(\mathcal{T}_{R}(M) \otimes_{R}^{\wedge}\right.\right. & \left.\left.N \otimes_{R}^{\wedge} \mathcal{T}_{R}(M)\right)\right) \\
& \stackrel{\simeq}{\rightarrow} \tilde{K}_{\mathcal{T}_{R}(M)}\left(\mathcal{T}_{R}(M) \ltimes\left(\mathcal{T}_{R}(M) \otimes_{R}^{\wedge} N \otimes_{R}^{\wedge} \mathcal{T}_{R}(M)\right)\right)
\end{aligned}
$$

described in section 4 of [DMcC1]. Following the description there, this homotopy equivalence is the stabilization of a given map

$$
\begin{aligned}
\bigvee_{Q \in \mathcal{P}_{\mathcal{T}_{R}(M)}} \operatorname{Hom}_{\mathcal{T}_{R}(M)}\left(Q, Q \otimes_{\mathcal{T}_{R}(M)} B .\left(\mathcal{T}_{R}(M) \otimes_{R}^{\wedge} N \otimes_{R}^{\wedge} \mathcal{T}_{R}(M)\right)\right) \\
\quad \rightarrow \bigvee_{Q \in \mathcal{P}_{\mathcal{T}^{(}(M)}} B .\left(\mathbf{1}_{Q}+I_{Q}\left(\mathcal{T}_{R}(M) \ltimes\left(\mathcal{T}_{R}(M) \otimes_{R}^{\wedge} N \otimes_{R}^{\wedge} \mathcal{T}_{R}(M)\right)\right)\right)
\end{aligned}
$$

which sends the 1-simplex corresponding to

$$
\begin{aligned}
\alpha: Q \rightarrow Q \otimes_{\mathcal{T}_{R}(M)}\left(\mathcal{T}_{R}(M) \otimes_{R}^{\wedge}\right. & \left.N \otimes_{R}^{\wedge} \mathcal{T}_{R}(M)\right) \\
& =Q \otimes_{\mathcal{T}_{R}(M)} B_{1}\left(\mathcal{T}_{R}(M) \otimes_{R}^{\wedge} N \otimes_{R}^{\wedge} \mathcal{T}_{R}(M)\right)
\end{aligned}
$$

to

$$
\begin{aligned}
\mathbf{1}_{Q}+\alpha \in \mathbf{1}_{Q}+I_{Q}\left(\mathcal{T}_{R}(M) \ltimes\left(\mathcal{T}_{R}(M) \otimes_{R}^{\wedge} N \otimes_{R}^{\wedge} \mathcal{T}_{R}(M)\right)\right. \\
\quad=B_{1}\left(\mathbf{1}_{Q}+I_{Q}\left(\mathcal{T}_{R}(M) \ltimes\left(\mathcal{T}_{R}(M) \otimes_{R}^{\wedge} N \otimes_{R}^{\wedge} \mathcal{T}_{R}(M)\right)\right) .\right.
\end{aligned}
$$

Therefore the loop corresponding to $\mathbf{1}_{P \otimes_{R} \mathcal{T}_{R}(M)}+\left(\mathbf{1}_{P \otimes_{R} \mathcal{T}_{R}(M)}-m\right)^{-1} \otimes n$ in $\tilde{K}_{\mathcal{T}_{R}(M)}\left(\mathcal{T}_{R}(M) \ltimes\left(\mathcal{T}_{R}(M) \otimes_{R}^{\wedge} N \otimes_{R}^{\wedge} \mathcal{T}_{R}(M)\right)\right)$ comes from

$$
\left(\mathbf{1}_{P \otimes_{R} \mathcal{T}_{R}(M)}-m\right)^{-1} \otimes n: P \otimes_{R} \mathcal{T}_{R}(M) \rightarrow \mathcal{T}_{R}(M) \otimes_{R}^{\wedge} N \otimes_{R}^{\wedge} \mathcal{T}_{R}(M) .
$$




\section{Lemma 4.2}

Proof: We look at the bisimplicial spectrum

$$
(p, q) \mapsto S^{\wedge p} \wedge X \wedge R^{\wedge q} \wedge S
$$

with the usual Hochschild-type face and degeneracy maps in both simplicial dimensions. Realizing first in the $p$-direction, we get that the realization of this bisimplicial set is

$$
\operatorname{THH}(R ; S \otimes \hat{S} X) \simeq \operatorname{THH}(R ; X) ;
$$

realizing first in the $q$-direction, we get that the realization is

$$
\operatorname{THH}\left(S ; X \otimes{ }_{R}^{\wedge} S\right) .
$$

\section{Lemma 4.3}

Proof: We will use the methods of [DMcC2]: we can model the product $\underline{S}^{\wedge p} \wedge$ $\underline{X} \wedge \underline{R}^{\wedge q} \wedge \underline{S}$ of the Eilenberg Mac Lane spectra associated to the simplicial rings and modules by

$$
\begin{gathered}
\operatorname{hocolim}_{\underline{X}} \underline{\operatorname{Map}}\left(S^{\sqcup \underline{X}}, \bigvee_{\underline{A}} \operatorname{Hom}_{S}\left(Q_{1}, Q_{0}\right)\left[S^{X_{0}^{1}}\right] \wedge \cdots \wedge \operatorname{Hom}_{S}\left(Q_{p}, Q_{p-1}\right)\left[S^{X_{p-1}^{1}}\right]\right. \\
\wedge \operatorname{Hom}_{R}\left(P_{0}, Q_{p} \otimes_{S} X\right)\left[S^{X_{p}^{1}}\right] \wedge \operatorname{Hom}_{R}\left(P_{1}, P_{0}\right)\left[S^{X_{0}^{2}}\right] \wedge \cdots \\
\left.\wedge \operatorname{Hom}_{R}\left(P_{q}, P_{q-1}\right)\left[S^{X_{q-1}^{2}}\right] \wedge \operatorname{Hom}_{S}\left(Q_{0}, P_{q} \otimes_{R} S\right)\left[S^{X_{q}^{2}}\right]\right)
\end{gathered}
$$

where $\underline{X}=\left(X_{0}^{1}, \ldots, X_{p}^{1}, X_{0}^{2}, \ldots, X_{q}^{2}\right)$ is a collection of finite sets and where

$$
\underline{A}=\left(Q_{0}, \ldots, Q_{p}, P_{0}, \ldots, P_{q}\right), Q_{i} \in \mathcal{P}_{S}, P_{i} \in \mathcal{P}_{R} .
$$

Boundary maps in this model come from the composition of maps, smashed with identity maps of a bimodule as needed, and the smashing together of spheres.

For the elements we need to represent, we can take $p=q=0$ and $X_{j}^{i}=\emptyset \forall i, j$, and look at elements in

$$
\operatorname{Hom}_{R}(P, Q \otimes S X) \wedge \operatorname{Hom}_{S}\left(Q, P \otimes_{R} S\right)
$$

for $P \in \mathcal{P}_{R}$ and $Q \in \mathcal{P}_{S}$.

Given a $P \in \mathcal{P}_{R}$ and an $R$-linear map $\alpha: P \rightarrow P \otimes_{R} X$ (where the $S-R$ bimodule $X$ is viewed as a left $R$-module through the unit map $R \rightarrow S$ ), we look at $Q=P \otimes_{R} S \in \mathcal{P}_{S}$. Since

$$
Q \otimes_{S} X=\left(P \otimes_{R} S\right) \otimes_{S} X \cong P \otimes_{R} X
$$


$\alpha$ can be viewed as an element of $\operatorname{Hom}_{R}\left(P, Q \otimes_{S} X\right)$. Consider

$$
\left(\alpha, \mathbf{1}_{Q}\right) \in \operatorname{Hom}_{R}\left(P, Q \otimes_{S} X\right) \wedge \operatorname{Hom}_{S}\left(Q, P \otimes_{R} S\right) .
$$

If we map $\underline{S}^{\wedge p} \wedge \underline{X} \wedge \underline{R}^{\wedge q} \wedge \underline{S} \rightarrow \operatorname{THH}\left(R ; X \otimes_{S} S\right)$, then $\left(\alpha, \mathbf{1}_{Q}\right)$ will be identified with the composition

$$
P \stackrel{\alpha}{\rightarrow} Q \otimes_{S} X \stackrel{1_{Q} \otimes_{S} \mathbf{1}_{X}}{\longrightarrow}\left(P \otimes_{R} S\right) \otimes_{S} X \cong P \otimes_{R} X
$$

that is, with $\alpha \in \operatorname{Hom}_{R}\left(P, P \otimes_{R} X\right)$. But if we map $\underline{S}^{\wedge p} \wedge \underline{X} \wedge \underline{R}^{\wedge q} \wedge \underline{S} \rightarrow$ $\operatorname{THH}\left(S ; X \otimes_{R}^{\wedge} S\right),\left(\alpha, \mathbf{1}_{Q}\right)$ will be identified with the composition

$$
Q \stackrel{1_{Q}}{\rightarrow} P \otimes_{R} S \stackrel{\alpha \otimes_{R} 1_{S}}{\longrightarrow}\left(Q \otimes_{S} X\right) \otimes_{R} S
$$

which is the map we called

$$
\alpha \otimes_{R}^{\wedge} \mathbf{1}_{S} \in \operatorname{Hom}_{S}\left(P \otimes_{R}^{\wedge} S, P \otimes_{R}^{\wedge} X \otimes_{R}^{\wedge} S\right) .
$$

This argument holds for any stage $n$ in the Waldhausen S-construction.

\section{REFERENCES}

B. S. Betley, Algebraic K-theory of parametrized endomorphisms, K-Theory $\mathbf{3 6}$ (2005), no. 3-4, 291-303.

BHM. M. Bökstedt, W.-C. Hsiang, I. Madsen, The cyclotomic trace and algebraic K-theory of spaces, Invent. Math. 111 (1993), no. 3, 465-539.

BS. S. Betley, C. Schlichtkrull, The cyclotomic trace and curves on K-theory, Topology 44 (2005), no. 4, 845-874.

CCGH. G. E. Carlsson, R. L. Cohen, T. Goodwillie, W.-C. Hsiang, The free loop space and the algebraic K-theory of spaces, $K$-Theory 1 (1987), no. 1, 53-82.

DGMcC. B. Dundas, T. Goodwillie, R. McCarthy, The Local structure of algebraic Ktheory, preprint of book.

DMcC1. B. Dundas, R. McCarthy, Stable K-theory and topological Hochschild homology, Ann. of Math. 140 (1994), 685-701.

DMcC2. B. Dundas, R. McCarthy, Topological Hochschild homology of ring functors and exact categories, J. Pure Appl. Algebra 109 (1996), no. 3, 231-294.

G. T. Goodwillie, Relative algebraic K-theory and cyclic homology, Ann. of Math. (2) 124 (1986), no. 2, 347-402.

G2. T. Goodwillie, Calculus II, Analytic functors, K-Theory 5 (1991/92), no. 4, 295-332. 
G3. T. Goodwillie, Calculus III, Taylor series, Geometry and Topology 7 (2003), 645-711.

Gr. D. Grayson, K-theory of endomorphisms, J. Algebra 48 (1977), 439-446.

H. L. Hesselholt, On the p-typical curves in Quillen's K-theory, Acta Math 177 (1996), $1-53$.

HM. L. Hesselholt, I. Madsen, On the K-theory of finite algebras over witt vectors of perfect fields, Topology 36 (1997), no. 1, 29-101.

I. Y. Iwachita, The Lefschetz-Reidemeister Trace in Algebraic K-theory, Ph. D. thesis, UIUC, 1999.

LMcC1. A. Lindenstrauss, R. McCarthy, The Taylor tower of the parametrized K-theory of endomorphisms, Geom. Topol., to appear.

LMcC2. A. Lindenstrauss, R. McCarthy, The algebraic K-theory of extensions of a ring by direct sums of itself, Indiana Univ. Math. J. 57 (2008), no. 2, 577-626.

McC. R. McCarthy, Relative algebraic K-theory and topological cyclic homology, Acta Math. 179 (1997), no. 2, 197-222.

M. J. Milnor, On the construction of FK, in J. F. Adams, Algebraic topology: a student's guide, London Mathematical Society Lecture Note Series 4, Cambridge University Press, London-New York, 1972.

NR. A. Neeman, A. Ranicki, Noncommutative localisation in algebraic K-theory. I, Geom. Topol 8 (2004), 1385-1425.

W1. F. Waldhausen, Algebraic K-theory of generalized free products. I, II, Ann. of Math. (2) 108 (1978), no. 1, 135-204.

W2. F. Waldhausen, Algebraic K-theory of spaces, in Algebraic and Geometric Topology (Rutgers 1983), 318-419, Lecture Notes in Math. 1126, Springer-Verlag Berlin-New York, 1985.

AYELET LindENSTRAUSS

alindens@indiana.edu

Department of Mathematics

Indiana University

Bloomington, IN 47405

U.S.A.

RANDY MCCARTHY rmccrthydilinois.edu

Department of Mathematics

University of Illinois at Urbana-Champaign

Urbana, IL 61801

U.S.A.

Received: November 17, 2010 